\title{
Comparative study using small-angle $x$-ray scattering and nitrogen adsorption in the characterization of silica xerogels and aerogels
}

\begin{abstract}
D. R. Vollet, D. A. Donatti, and A. Ibañez Ruiz
Department of Physics, IGCE-UNESP, P.O. 178, CEP 13500-970 Rio Claro, São Paulo, Brazil

(Received 6 August 2003; revised manuscript received 11 November 2003; published 27 February 2004)

A comparative study using small-angle x-ray scattering (SAXS) and nitrogen adsorption has been carried out in the structural characterization of silica xerogels and aerogels, obtained from tetraethoxysilane sonohydrolysis. The specific surface and the mean pore size as measured by both the techniques were found to be in notable agreement in all cases for aerogels and xerogels. According to the SAXS data, aerogels at $500{ }^{\circ} \mathrm{C}$ exhibit a mass fractal structure with fractal dimension $D \sim 2.4$ in the range between the correlation length $\xi \sim 5.3 \mathrm{~nm}$ and $a \sim 0.75 \mathrm{~nm}$. An experimental method to probe the mass fractal structure of aerogels from exclusively nitrogen adsorption isotherms has been presented. For aerogels at $500^{\circ} \mathrm{C}$, we have found $D \sim 2.4$ in the range between the pore width $2 r_{\xi} \sim 33 \mathrm{~nm}$ and $2 r_{\mathrm{a}} \sim 4.5 \mathrm{~nm}$, which is in notable agreement with the SAXS results $(D$ $\sim 2.4, \xi \sim 5.3 \mathrm{~nm}, a \sim 0.75 \mathrm{~nm}$ ) if we assign the pore width $2 r$ probed by the Kelvin equation in the adsorption method to the Bragg distance $2 \pi / q$ associated to the correlation length $1 / q$ probed by SAXS.
\end{abstract}

DOI: 10.1103/PhysRevB.69.064202

PACS number(s): 61.10.Eq, 61.43.Hv, 81.20.Fw, 82.70.Gg

\section{INTRODUCTION}

A large variety of glass and glass ceramics has been obtained by sol-gel process from the hydrolysis of tetraethoxysilane (TEOS). ${ }^{1}$ Since water and TEOS are immiscible, sonochemistry $^{2}$ is an effective method to promote hydrolysis in presence of the acid catalyst to avoid the addition of a mutual solvent such as ethanol, as in the conventional method, since ethanol is a product of both the hydrolysis and the alcohol producing condensation reactions.

Wet gels frequently exhibit structures with mass fractal features consisting of a continuous solid network imbibed in a liquid phase, which can occupy until about $95 \%$ of the volume sample. Supercritical drying (aerogels) and evaporation drying (xerogels) are usual methods in dried gel production. The structure of aerogels are often very similar to that of the original aged wet gels, ${ }^{3}$ except by the contrast variation resulting from the liquid phase removal, since the absence of capillary forces in the supercritical process allows most of the original structure of the solid network to be preserved. On the other hand, the structure of xerogels is profoundly modified on the liquid phase removal due to the capillary forces acting on the solid network under the evaporation process. ${ }^{4}$ Heat treatment imposes further structural evolutions on both xerogels ${ }^{5}$ and aerogels. ${ }^{6}$

In general, different structures for gels have been reported depending on the starting materials, the initial conditions of preparation as $p \mathrm{H}$, alcoxide/water molar ratio, type of catalyst, temperature and method employed for hydrolysis (ultrasound or conventional), and also on the conditions of gelification and subsequent treatments for aging and drying of the gels. Even different techniques employed in the gel structural characterization applied to the same sample may lead to structures of discussible similarity and, not always, a clear correlation between the structural parameters probed by different techniques may be unequivocally established.

In this work, the structural characteristics of xerogels and aerogels, obtained from sonohydrolysis of TEOS, were stud- ied by means of small-angle x-ray scattering (SAXS) and nitrogen adsorption. The structural parameters as determined by both techniques are shown to be in agreement in all the cases. In addition, a method to probe the mass fractal properties of aerogels exclusively from nitrogen adsorption isotherms is presented and shown to be in reasonable agreement with the results of the mass fractal analysis as determined by SAXS.

\section{EXPERIMENT}

The samples were prepared from the sonohydrolysis of mixtures of $25 \mathrm{ml}$ of tetraethoxysilane (TEOS), $8 \mathrm{ml}$ of distilled and dionized water, and $5 \mathrm{ml}$ of $0.1 \mathrm{~N} \mathrm{HCl}$ as a catalyst. The resulting $p \mathrm{H}$ of the mixture was about $1.5-2.0$. The hydrolysis was promoted during 10 minutes under a constant power $(60 \mathrm{~W})$ of ultrasonic radiation. Next, the sol was diluted in $14.3 \mathrm{ml}$ of water and sonication was continued for 2 min for complete homogenization. The $p \mathrm{H}$ of the resulting sol was adjusted to 4.5 by addition of $\mathrm{NH}_{4}(\mathrm{OH})$. The final water/TEOS molar ratio was equal to 14.4 . The resulting sol was cast in sealed containers and kept under saturated conditions for 20 days at $40^{\circ} \mathrm{C}$ for gelation and aging. Xerogels were obtained from slow heating of the gels up to $500^{\circ} \mathrm{C}$. Aerogels were obtained by a two step process: first, the liquid phase of the wet sonogels was exchanged by pure ethanol at room temperature, changing the liquid volume (about 10 times the gel volume) each $24 \mathrm{~h}$ during 10 days; second, ethanol was exchanged by liquid $\mathrm{CO}_{2}$ in an autoclave followed by supercritical $\mathrm{CO}_{2}$ extraction. Samples of aerogels were heat treated at temperature of $500{ }^{\circ} \mathrm{C}$ and $900{ }^{\circ} \mathrm{C}$ under atmospheric conditions for about 10 hours. Xerogels and aerogels were studied by means of SAXS and nitrogen adsorption techniques.

The SAXS experiments were carried out using synchrotron radiation with a wavelength $\lambda=0.1608 \mathrm{~nm}$. The beam was monochromatized by a silicon monochromator and collimated by a set of slits defining a pin-hole geometry. A one-dimensional position sensitive $\mathrm{x}$-ray detector was used 
to record the SAXS intensity $I(q)$ as function of the modulus of the scattering vector $q=(4 \pi / \lambda) \sin (\theta / 2)$, where $\theta$ is the scattering angle. The experimental set allowed to get SAXS data from $q_{0}=0.19 \mathrm{~nm}^{-1}\left(q_{0}=0.10 \mathrm{~nm}^{-1}\right.$ in a set of data $)$ to $q_{\mathrm{m}}=4.4 \mathrm{~nm}^{-1}$ with a resolution of $\Delta q=3.36$ $\times 10^{-3} \mathrm{~nm}^{-1}$. The data were corrected by the parasitic scattering and the sample attenuation, and normalized by the intensity of the incident beam and the logarithm of the sample attenuation.

Nitrogen adsorption isotherms were obtained at liquid nitrogen temperature using a commercial pore and surface analyzer apparatus (ASAP 2010 Micromeritics). The data were analyzed for BET specific surface $\left(S_{\mathrm{BET}}\right)$, total pore volume per mass unit $V_{\mathrm{p}}$ through the total adsorbed volume of nitrogen at a single point close to the saturation pressure, micropore volume $V_{\text {micro }}$ through $t$-plot method, ${ }^{7}$ mean pore size $\left(l_{\mathrm{BET}}=4 V_{\mathrm{p}} / S_{\mathrm{BET}}\right)$, and pore volume distribution, as determined by using the classical Kelvin equation and the Harkins and Jura model for cylindrical pores. ${ }^{7}$

\section{INTERPRETATION OF THE SAXS DATA}

Aerogels frequently exhibit mass fractal structures that can be recognized by typical power-law decrease on $q$ of the SAXS intensity in a $q$ range between $\xi \gg q^{-1} \gg a$, where $\xi$ is the characteristic length of the fractal structure and $a$ the characteristic length of the primary particles composing the structure. ${ }^{8}$ The SAXS intensity departs from the power-law regime at low- $q$ values due to the finite correlation range $\xi$ of the mass fractal structure, and at high- $q$ values due to the characteristics of the primary particle composing the structure.

For aerogels, we first analyzed the SAXS intensity at the low- and medium- $q$ regions ${ }^{9}$ in which it can be fitted by

$$
\begin{aligned}
I(q)= & A \sin [(D-1) \arctan (q \xi)] /\left(1+q^{2} \xi^{2}\right)^{(D-1) / 2}(D \\
& -1) q \xi,
\end{aligned}
$$

where $D$ is the mass fractal dimension of the structure, a physically acceptable value between $1<D<3$, and $A$ is a constant for a given $D$ and $\xi$. At high- $q$ region, it was found that the intensity crossing over to power law decrease on $q$ as

$$
I(q) \sim q^{-\alpha},
$$

with the exponent $\alpha$ found quite close to 4 . The values expected for $\alpha$ for surface fractal structures are between 3 and 4 , so the surface fractal dimension $D_{\mathrm{S}}$ is given ${ }^{8}$ by

$$
D_{\mathrm{S}}=6-\alpha \quad(3<\alpha<4) .
$$

A system of particles with perfectly smoothed surface has $D_{S}$ $=2$, so Eq. (2) becomes the classical Porod's law ${ }^{10}$ cast as

$$
I(q) \sim q^{-4} .
$$

The surface per unit volume $(S / V)$ of a porous system obeying Porod's law can be determined from SAXS through the equation

$$
(S / V)=\pi \phi(1-\phi) K_{\mathrm{P}} / Q,
$$

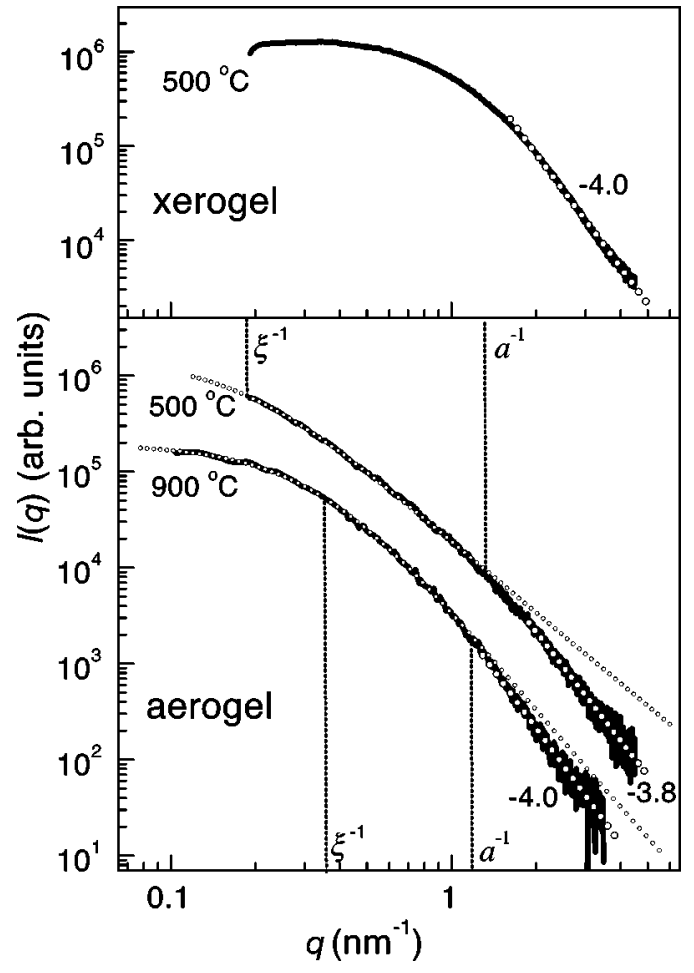

FIG. 1. SAXS intensity as a function of the modulus of scattering vector $q$ for xerogels and aerogels. The medium circle lines are linear fitting of Eq. (2) to the experimental data at high $q$ in Porod's region and the slopes of which are indicated in each case. The small circle lines drawn for aerogels are the fitting of the mass fractal approach [Eq. (1)] to the experimental data at low- and medium- $q$ regions.

where $\phi$ is the pore volume fraction, $K_{\mathrm{P}}$ is the constant of Porod's law defined as

$$
K_{\mathrm{P}}=\lim _{q \rightarrow \infty} I(q) q^{4},
$$

and $Q$ is the integral, known as invariant of the system, given by

$$
Q=\int_{0}^{\infty} q^{2} I(q) d q
$$

In addition, the pore mean size $l_{\text {SAXS }}$ can be obtained from $(S / V)$ through the equation

$$
l_{\mathrm{SAXS}}=4 \phi /(S / V)
$$

and the SAXS specific surface $S_{\text {SAXS }}$ through

$$
S_{\mathrm{SAXS}}=(1 / \rho)(S / V)
$$

where $\rho$ is the sample bulk density.

\section{RESULTS}

Figure 1 shows the curves of the SAXS intensity $I(q)$ as a function of the modulus of scattering vector $q$ for xerogel treated at $500^{\circ} \mathrm{C}(\mathrm{X} 500)$ and for samples of aerogels treated at $500{ }^{\circ} \mathrm{C}(\mathrm{A} 500)$ and $900{ }^{\circ} \mathrm{C}(\mathrm{A} 900)$. Figure 2 shows the 


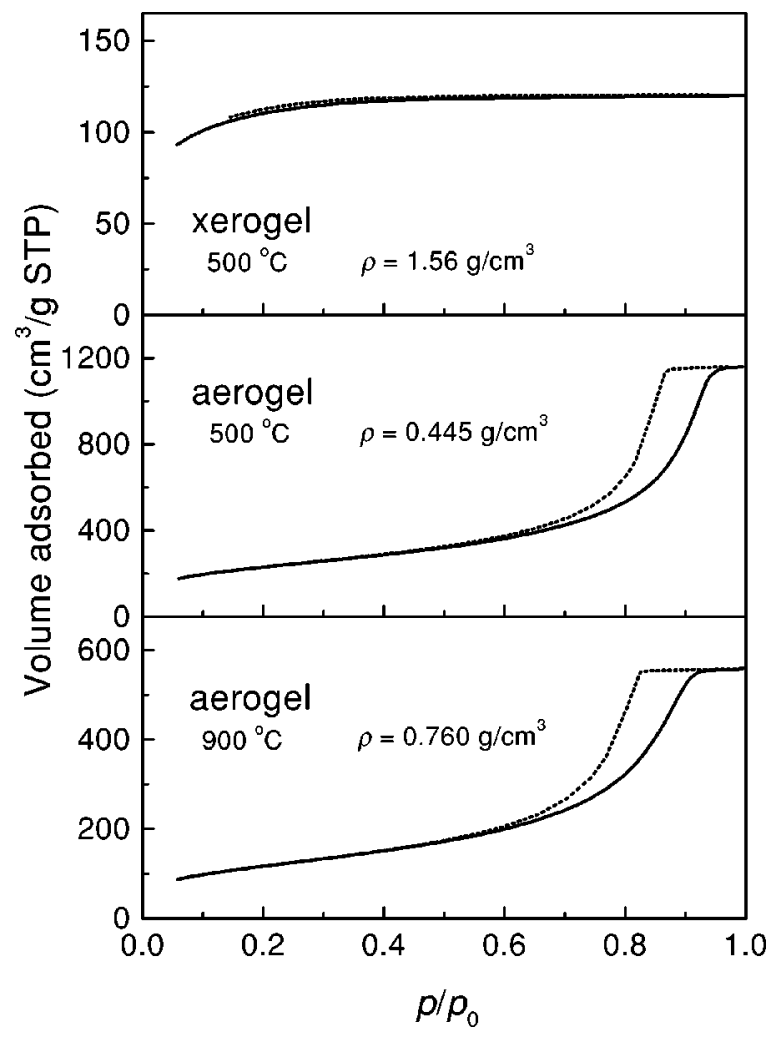

FIG. 2. Nitrogen adsorption isotherms carried out at liquid nitrogen temperature for xerogels and aerogels. The dotted lines correspond to the desorption branch. The bulk density $\rho$ is indicated in each case.

nitrogen adsorption isotherms as obtained at liquid nitrogen temperature for xerogels and aerogels. Xerogels exhibit isotherms that are more properly classified as type I while aerogels as type IV according to International Union of Pure and Applied Chemistry (IUPAC) classification. ${ }^{11}$

The scattering from the xerogel X500 and the aerogel A900 show classical features of a two-phase system with the asymptotic approximation at high- $q$ given by Porod's law $I(q) \sim q^{-4}$. The scattering at high- $q$ from the aerogel A500 decays with an exponent $\alpha=3.8 \pm 0.1$ revealing a small roughness associated to the surface, which would be fractal with dimensionality $D_{\mathrm{S}}=2.2 \pm 0.1$. Table I shows the SAXS

TABLE I. Structural parameters as determined by SAXS and nitrogen adsorption isotherms for xerogels and aerogels.

\begin{tabular}{lccc}
\hline \hline & \multicolumn{2}{c}{ Aerogel } & Xerogel \\
& $500{ }^{\circ} \mathrm{C}$ & $900^{\circ} \mathrm{C}$ & $500^{\circ} \mathrm{C}$ \\
\hline$S_{\text {SAXS }}\left(10^{2} \mathrm{~m}^{2} / \mathrm{g}\right)$ & $8.1 \pm 0.2$ & $4.1 \pm 0.2$ & $3.9 \pm 0.2$ \\
$S_{\text {BET }}\left(10^{2} \mathrm{~m}^{2} / \mathrm{g}\right)$ & $8.32 \pm 0.08$ & $4.28 \pm 0.04$ & $3.85 \pm 0.08$ \\
$l_{\text {SAXS }}(\mathrm{nm})$ & $8.9 \pm 0.4$ & $8.5 \pm 0.4$ & $1.9 \pm 0.1$ \\
$l_{\text {BET }}(\mathrm{nm})$ & $8.6 \pm 0.1$ & $8.1 \pm 0.1$ & $1.9 \pm 0.1$ \\
$V_{\mathrm{p}}\left(\mathrm{cm}^{3} / \mathrm{g}\right)$ & $1.793 \pm 0.004$ & $0.862 \pm 0.002$ & $0.186 \pm 0.001$ \\
$V_{\text {micro }}\left(\mathrm{cm}^{3} / \mathrm{g}\right)$ & $0.021 \pm 0.001$ & $\sim 0$ & $0.082 \pm 0.001$ \\
$\rho\left(\mathrm{g} / \mathrm{cm}^{3}\right)$ & $0.445 \pm 0.002$ & $0.760 \pm 0.002$ & $1.56 \pm 0.01$ \\
$\phi$ & $0.798 \pm 0.004$ & $0.655 \pm 0.003$ & $0.290 \pm 0.002$ \\
\hline \hline
\end{tabular}

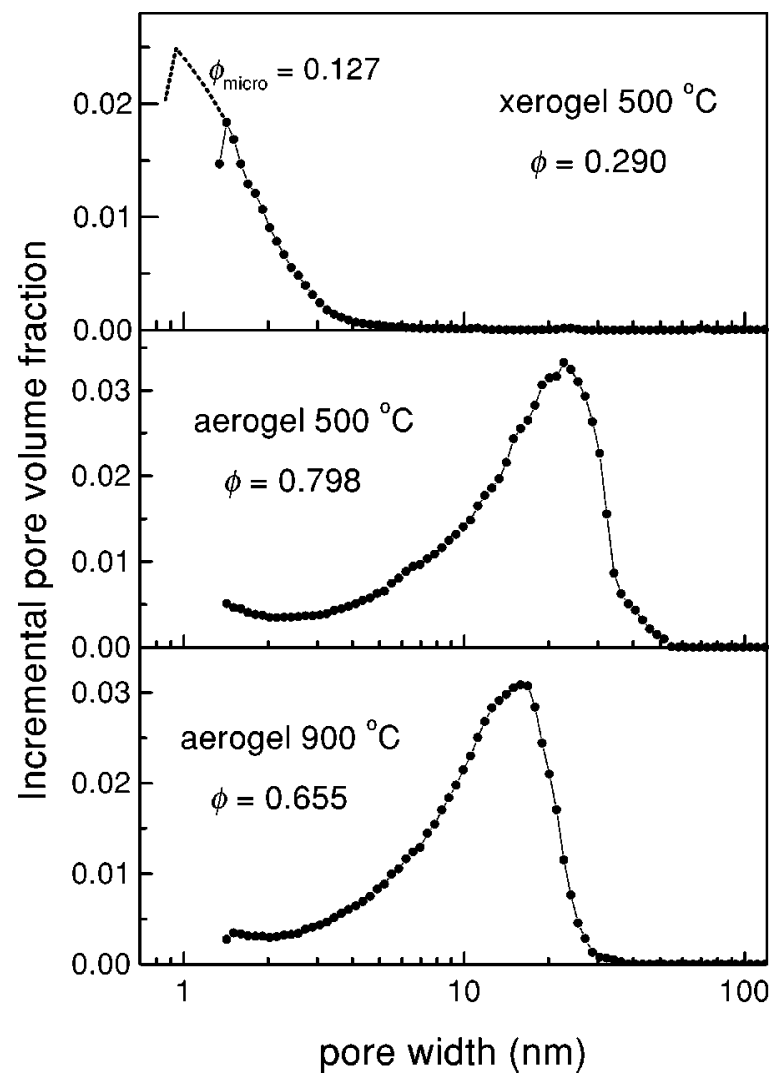

FIG. 3. Pore volume distribution as obtained by the Kelvin equation and the classical Harkins and Jura model for cylindrical pores from the nitrogen adsorption isotherms. The total pore volume fraction $\phi$ is indicated in each case. The total micropore volume fraction $\phi_{\text {micro }}$ associated to the xerogel, as determined by $t$-plot method, has been distributed as shown in the figure by linear extrapolation from the Harkins and Jura mesopore distribution.

structural parameters $S_{\mathrm{SAXS}}$ and $l_{\mathrm{SAXS}}$ as determined through Eqs. (8) and (9) compared to $S_{\mathrm{BET}}$ and $l_{\mathrm{BET}}$ as determined by nitrogen adsorption for aerogels and xerogels. The results as determined by both the techniques are in excellent agreement in all the cases. The minor contribution to the scattering at high $q$ due to the small roughness of the surface associated to the aerogel A500 has been subtracted, following standard methods ${ }^{10}$ in the obtaining of $K_{\mathrm{P}}$ and then $S_{\mathrm{SAXS}}$. So the value of $S_{\mathrm{SAXS}}$ as determined for the aerogel A500 should be interpreted as a minimum value.

In Table I, the bulk density $\rho$ and the pore volume fraction $\phi$ have been evaluated from the total pore volume per mass unit $V_{\mathrm{p}}$ through the relations $(1 / \rho)=\left(1 / \rho_{\mathrm{a}}\right)+V_{\mathrm{p}}$ and $\phi$ $=\rho V_{\mathrm{p}}$, respectively, assuming the value $2.2 \mathrm{~g} / \mathrm{cm}^{3}$ for the skeletal density $\rho_{\mathrm{a}}$, as frequently quoted for fused silica. Xerogels show an appreciable quantity of micropores $\left(V_{\text {micro }}\right)$, pores with size minor than about $1.4 \mathrm{~nm}$, while aerogels exhibit only a negligible quantity of micropores at $500^{\circ} \mathrm{C}$ and nothing at $900^{\circ} \mathrm{C}$, as measured by $t$ plots from the adsorption isotherms. In the total pore volume $V_{\mathrm{p}}$ in Table I is included the quantity $V_{\text {micro }}$.

Figure 3 shows the incremental pore volume fraction for xerogels and aerogels according to the pore volume distribution as obtained from the adsorption isotherms. The pore 
TABLE II. Mass fractal structural parameters for aerogels as determined by SAXS and from the nitrogen adsorption isotherms.

\begin{tabular}{lccccc}
\hline \hline Technique & Temperature & $D$ & $\xi(\mathrm{nm})$ & $a(\mathrm{~nm})$ & $\xi / a$ \\
\hline SAXS & $500^{\circ} \mathrm{C}$ & $2.40 \pm 0.02$ & $5.26 \pm 0.04$ & $0.75 \pm 0.05$ & $7.1 \pm 0.6$ \\
$\mathrm{~N}_{2}$ adsorption & $500^{\circ} \mathrm{C}$ & $2.38 \pm 0.02$ & $5.3 \pm 0.1$ & $0.7 \pm 0.1$ & $7 \pm 1$ \\
$\mathrm{SAXS}$ & $900^{\circ} \mathrm{C}$ & $2.88 \pm 0.06$ & $2.84 \pm 0.08$ & $1.1 \pm 0.1$ & $2.6 \pm 0.3$ \\
$\mathrm{~N}_{2}$ adsorption & $900^{\circ} \mathrm{C}$ & $2.42 \pm 0.02$ & $3.6 \pm 0.1$ & $0.8 \pm 0.1$ & $4.8 \pm 0.5$ \\
\hline \hline
\end{tabular}

width corresponds to the quantity $d=2 r$, where $r$ is the radius of the pore, assumed as cylindrical in the Harkins and Jura model. The total pore volume fraction $\phi=\rho V_{\mathrm{p}}$ is shown in Fig. 3 in each case. The pore volume of the xerogel increases from a maximum pore width of about $d \sim 5.0 \mathrm{~nm}$ in direction to the micropore region. The total micropore volume fraction $\phi_{\text {micro }}=0.127$ (as determined by $t$ plot) associated to the xerogel has been distributed as shown in Fig. 3 by linear extrapolation from the Harkins and Jura mesopore distribution. The pore volume of aerogels increases from the micropore region to the mesopore region until it reaches a maximum value, occurring at a pore-width $d \sim 25 \mathrm{~nm}$ at $500{ }^{\circ} \mathrm{C}$ and $d \sim 18 \mathrm{~nm}$ at $900^{\circ} \mathrm{C}$, and sharply drop again going to zero at a maximum pore width $d_{\max }$, which was found to be $\sim 50 \mathrm{~nm}$ at $500{ }^{\circ} \mathrm{C}$ and $\sim 30 \mathrm{~nm}$ at $900{ }^{\circ} \mathrm{C}$.

An independent measurement of the bulk density for these aerogels was carried out by mercury displacement in Ref. 6 . We have found $\rho=(0.43 \pm 0.02) \mathrm{g} / \mathrm{cm}^{3}$ for A500 and $\rho$ $=(0.76 \pm 0.02) \mathrm{g} / \mathrm{cm}^{3}$ for A900, in good agreement with the corresponding values of Table I as measured from the total adsorbed volume of nitrogen $\left(V_{\mathrm{p}}\right)$. Although the SAXS measurements were performed on monolithic aerogel slices, another independent bulk density measurement could not be obtained from these data since the SAXS intensity has not been obtained in absolute units. It has been pointed ${ }^{12,13}$ that, in the case of low density aerogels, the adsorption method applies stress on the sample during measurement leading to an underestimation of pore volume. This seems not to be the case for our samples of aerogels produced from sonogels due to the good agreement found between the bulk density values as evaluated from both the mentioned independent methods. This may be due to the value not too low for the density of our sonogel-derived aerogels. It has been pointed ${ }^{14}$ that when the bulk density exceeds $0.38 \mathrm{~g} / \mathrm{cm}^{3}$ the values for the specific surface areas obtained from both BET and SAXS are close.

\section{DISCUSSION}

Aerogels exhibit a particularly interesting pore structure since an evaluation of the incremental surface area from the Harkins and Jura pore volume distribution shown in Fig. 3 yields an almost constant value in the range of pore width from $\sim 3 \mathrm{~nm}$ up to $\sim 25 \mathrm{~nm}$ at $500{ }^{\circ} \mathrm{C}$, and up to $\sim 17 \mathrm{~nm}$ at $900^{\circ} \mathrm{C}$. Evidently, a fraction of the pore volume is eliminated when the aerogel is heated from $500^{\circ} \mathrm{C}$ to $900^{\circ} \mathrm{C}$.

We wonder if such a particular structure could be associated to a mass fractal structure. We first analyzed the SAXS intensity from the aerogels by fitting Eq. (1) to the experimental A500 and A900 data. Figure 1 (bottom) shows the good fitting of Eq. (1) to the experimental data at low- and medium- $q$ regions for the aerogels and a crossover at about $q_{\mathrm{a}} \sim a^{-1}$ in Porod's region. The SAXS structural parameters $D, \xi, a$, and the evaluated ratio $\xi / a$, are shown in Table II together with the corresponding values as obtained from the pore volume distribution from the nitrogen adsorption isotherms, as discussed ahead.

The value $D=2.40 \pm 0.02$, as obtained by SAXS for the aerogel A500 in a fractal range of almost one order of magnitude for the ratio $\xi / a(\xi / a \sim 7)$, is in good agreement with the results obtained by Vacher et al., ${ }^{9}$ who have found $D$ $=2.40 \pm 0.03$ for a great variety of acid-catalyzed prepared silica aerogels. For the aerogel A900, the range of fractality as determined by SAXS was found to be very small $(\xi / a$ $\sim 2.6$ ). As postulated by Woignier et al., ${ }^{15}$ systems with reasonable fractal characteristics must show a fractal behavior over more than one order of magnitude for the ratio $\xi / a$, or $\xi / a>10$. Then, the value $D \sim 2.9$ as obtained by SAXS for the aerogel A900 should be interpreted as a very critical value due to the small mass fractal range as found by fitting the SAXS data $(\xi / a \sim 2.6)$. However, there may be a more fundamental reason for obtaining such a high value for $D$ by using Eq. (1).

We imagined a way to generate a mass fractal structure from the experimental pore volume distribution, as determined from the nitrogen adsorption isotherms, employing a method in analogy to that used to generate nonrandom fractals, starting from an initial configuration by a sequence of approximants. ${ }^{16}$ Experimentally, we start from a homogeneous solid with density $\rho_{\mathrm{a}}$, equal to that of fused silica $\left(\sim 2.2 \mathrm{~g} / \mathrm{cm}^{3}\right)$, and follow incorporating to the structure, step by step, each incremental pore volume per mass unit $\delta V_{\mathrm{p}}\left(r_{\mathrm{i}}\right)$ corresponding to the pores with radius $r_{\mathrm{i}}$, starting from $r_{\mathrm{i}}=0$ up to $r_{\mathrm{i}}=r$, and probing the evolution of the resulting bulk density $\rho(r)$. The process can be cast as

$$
\frac{1}{\rho(r)}=\frac{1}{\rho_{\mathrm{a}}}+\sum_{r_{\mathrm{i}}=0}^{r_{\mathrm{i}}=r} \delta V_{\mathrm{p}}\left(r_{\mathrm{i}}\right) .
$$

Figure 4 shows $\rho(r)$ for the aerogels, in a log-log scale with the structure scale length as probed by the pore width $2 r$, as evaluated through Eq. (10) from the pore volume distribution shown in Fig. 3. Accordingly, the density $\rho(r)$ tends to a constant value equal to the bulk density $\rho$ as the pore width $2 r$ increases up to its maximum value in each case. So, the bulk density $\rho$ could be assigned to $\rho_{\xi}$. The value of pore 


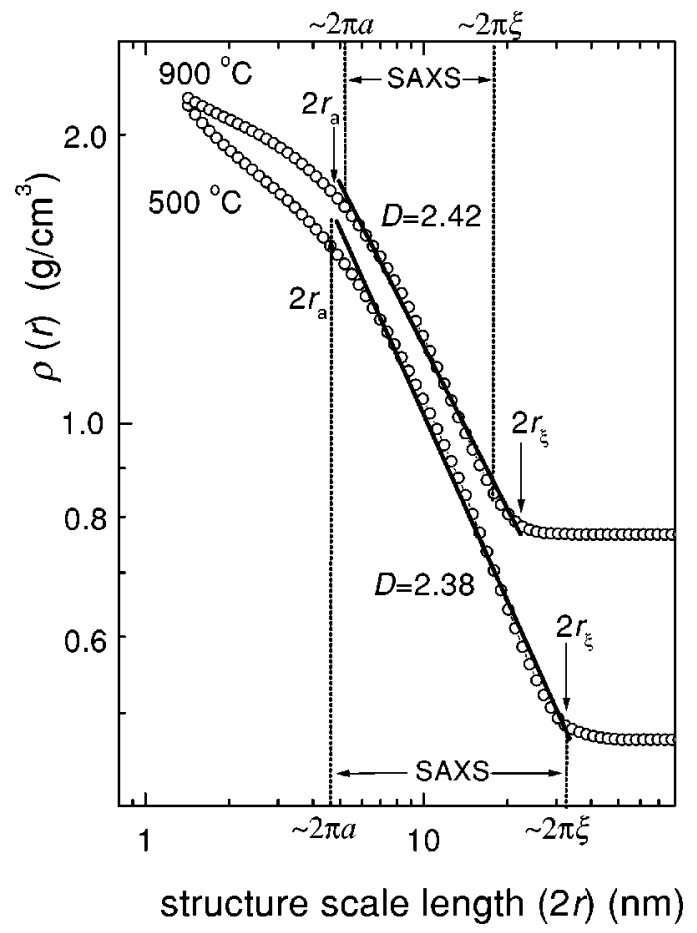

FIG. 4. Step by step evaluation of the bulk density $\rho(r)$ of the aerogels from the pore volume distribution through the method described by Eq. (10). The straight lines are linear fittings of Eq. (12) in the fractal range between the pore width $2 r_{\xi}$ and $2 r_{\mathrm{a}}$. The slope of straight lines should be $D-3$. The Bragg distances $2 \pi \xi$ and $2 \pi a$ associated to the correlation length $1 / q$ probed by SAXS ( $\xi$ and $a$ ) are shown in order to a direct comparison.

width $2 r$ from which $\rho(r)$ becomes practically equal to the bulk density was found quite close to the value $2 \pi \xi$, the Bragg distance associated to the correlation length $\xi$ as probed by SAXS. The agreement is notable for the aerogel A500 for which $\xi / a \sim 7$. Since both the techniques have yielded the same structural parameters as shown in Table I, then the pore width $2 r$ probed by the Kelvin equation in the adsorption experiment is more properly assigned to the Bragg distance associated to the correlation length $1 / q$ ( $\xi$ or a) probed by SAXS. The Bragg distance range associated to the correlation lengths $\xi$ and $a$ as determined by SAXS is shown in Fig. 4 for both the aerogels for a direct comparison. A clear inflection point in $\rho(r)$ was not found in the micropore region accounting for the constancy of $\rho(r)$ for $2 r$ $<\sim 2 \pi a$ when $\rho(r)=\rho_{\mathrm{a}}$. It is probably due to the micropore contribution, which has been added together in the evaluation of Eq. (10).

For a mass fractal structure the bulk density $\rho_{\xi}$ can be cast ${ }^{15}$ as

$$
\rho_{\xi}=\rho_{\mathrm{a}}(\xi / a)^{D-3},
$$

so the evaluated $\rho(r)$ in the range $a \leqslant r \leqslant \xi$ should be

$$
\rho(r)=\rho_{\mathrm{a}}(r / a)^{D-3} .
$$

Figure 4 shows that Eq. (12) fits reasonably well to the experimental $\rho(r)$ in an intermediary interval of pore width, since the plot of Eq. (12) in a log-log scale with the pore width $2 r$ should be a straight line with slope $D-3$. Then, we have an alternative method to determine the mass fractal dimension $D$ of aerogels. Table II shows the values of $D$ and the range of fractality associated to the pore width $2 r_{\xi}$ $\sim 2 \pi \xi$ and $2 r_{\mathrm{a}} \sim 2 \pi a$ as determined by nitrogen adsorption compared to the SAXS ones. The value $D \sim 2.4$ and the mass fractal range $\xi / a \sim 7$ as measured by both the techniques are in notable agreement for the aerogel at $500^{\circ} \mathrm{C}$. The value $D \sim 2.4$ as measured by nitrogen adsorption is in agreement for both the aerogels A500 and A900. So, the method to probe the mass fractal characteristics from the pore volume distribution by nitrogen adsorption through Eqs. (10) and (12) seems to be proper in studying aerogels.

The obtaining of the fractal dimension $D$ from the pore size distribution has been first presented in a different way by Pfeifer and Avnir ${ }^{17}$ and the method has been applied to aerogels. ${ }^{18-20}$ The method is based on the slope behavior of the pore size distribution curve which is related to the fractal dimension $D$ by the relation ${ }^{17}$

$$
-\frac{d V(r)}{d r} \propto r^{2-D}
$$

where $V(r)$ is the total volume of pores of size larger than $r$. Then we have $V(r)=V_{\mathrm{p}}-\sum_{r_{\mathrm{i}}=0}^{r_{\mathrm{i}}=r} \delta V_{\mathrm{p}}\left(r_{\mathrm{i}}\right)$, where the sum was defined in Eq. (10). By subtracting from Eq. (10) the relation $(1 / \rho)=\left(1 / \rho_{\mathrm{a}}\right)+V_{\mathrm{p}}$, which we have used in obtaining the bulk density, bearing in mind that $\rho=\rho_{\xi}$, we obtain

$$
\frac{1}{\rho(r)}=\frac{1}{\rho_{\xi}}-V(r)
$$

and, using Eq. (12),

$$
\frac{1}{\rho_{\mathrm{a}}}(r / a)^{3-D}=\frac{1}{\rho_{\xi}}-V(r) .
$$

The derivation of Eq. (15) with respect to $r$ yields Eq. (13). Then the method employed in this work in determining $D$ is equivalent to that due to Pfeifer and Avnir, except that Eq. (10) is an integrated form of Eq. (13) obtained from completely independent assumptions.

However, the application of Eq. (13) to our pore size distribution curves yields values for $D$ which are minor than 2 ( $D=1.84$ for A500 and $D=1.68$ for A900), which are not in agreement with the SAXS results. Surprisingly, the value of $D$ in the aerogel A900 (clearly more compacted) was found smaller than that in A500 when analyzed from Eq. (13). The reason for this remains not quite clear yet. It may be associated to the effects of the lower and upper cutoff of the fractal range which makes Eq. (13) not quite convenient to determine $D .{ }^{18}$ It has been pointed ${ }^{18,21}$ that, for larger $D$, Eq. (13) appears to underestimate real values for $D$ in aerogels, and the mass fractal dimension characteristics obtained from adsorption method appear to differ from those determined from SAXS or small-angle neutron scattering (SANS). However, there may be a more fundamental reason for obtaining different values for $D$ by employing clearly equivalent methods. For instance, the assumption of cylindrical pores of various radii $r$ and lengths $h$, frequently assumed in the obtaining of 
the pore size distribution, bring no additional problem with the incremental adsorbed volume, since the adsorbed volume is a direct measure in the adsorption method. Then, Eq. (10) is independent of how $h$ is a function of $r$. The explicit dependence on $r$ comes from the application of the scaling law of the density according to the mass fractal characteristics given by Eq. (12). On the other side, the derivation of $V(r)$ with respect to $r$ may hide an implicit dependence on $h$ that might be responsible for the different probing on applying both the methods.

\section{CONCLUSIONS}

Xerogels at $500{ }^{\circ} \mathrm{C}$ present most of the pore volume composed by pores with pore width smaller than $\sim 5.0 \mathrm{~nm}$ and pore mean size of $\sim 1.9 \mathrm{~nm}$, while aerogels show a pore volume distribution with a maximum point at a pore-width of $\sim 25 \mathrm{~nm}$ and pore mean size of $\sim 8.6 \mathrm{~nm}$. The last values diminishing to $\sim 18 \mathrm{~nm}$ and $\sim 8.1 \mathrm{~nm}$, respectively, by heating the aerogel up to $900^{\circ} \mathrm{C}$.

The specific surface and the mean pore size as measured by SAXS and nitrogen adsorption techniques were found to be in notable agreement in all cases for xerogels and aerogels. According to the SAXS results, aerogel at $500^{\circ} \mathrm{C}$ exhibits a mass fractal structure with fractal dimension $D$ $\sim 2.4$ in a fractal range between the correlation length $\xi$ $\sim 5.3 \mathrm{~nm}$ and $a \sim 0.75 \mathrm{~nm}$. At $900^{\circ} \mathrm{C}$, the range of fractality is shortened by pore elimination so that the determination of $D$ becomes very critical by SAXS.

An experimental method to probe the mass fractal structure from the pore volume distribution obtained exclusively from the nitrogen adsorption isotherm has been applied to the aerogels. For the aerogel at $500{ }^{\circ} \mathrm{C}$, we have obtained $D \sim 2.4$ in the fractal range between the pore width $2 r_{\xi}$ $\sim 33 \mathrm{~nm}$ and $2 r_{\mathrm{a}} \sim 4.5 \mathrm{~nm}$, in notable agreement with the SAXS results $(D \sim 2.4, \xi \sim 5.3 \mathrm{~nm}, a \sim 0.75 \mathrm{~nm})$ if we assign the pore width $2 r$ probed by the Kelvin equation in the adsorption method to the Bragg distance $2 \pi / q$ associated to the correlation length $1 / q$ ( $\xi$ or $a$ ) probed by SAXS. The method of the mass fractal analysis from nitrogen adsorption isotherms has equally been applied to the aerogel at $900{ }^{\circ} \mathrm{C}$ in a minor fractal range yielding a values lightly greater than $D \sim 2.4$.

The method to probe the mass fractal characteristics from the pore volume distribution obtained from the nitrogen adsorption isotherms seems proper in studying aerogels.

\section{ACKNOWLEDGMENTS}

Research was partially supported by LNLS-National Synchrotron Light Laboratory (Brazil) and FAPESP (Brazil).
${ }^{1}$ C. J. Brinker and G. W. Scherer, Sol-Gel Science: The Physics and Chemistry of Sol-Gel Processing (Academic, San Diego, 1990).

${ }^{2}$ M. Tarasevich, Am. Ceram. Soc. Bull. 63, 500 (1984).

${ }^{3}$ D.A. Donatti, D.R. Vollet, and A. Ibañez Ruiz, J. Non-Cryst. Solids 292, 44 (2001).

${ }^{4}$ G.W. Scherer, J. Non-Cryst. Solids 100, 72 (1988).

${ }^{5}$ D.R. Vollet, D.A. Donatti, and A. Ibañez Ruiz, J. Non-Cryst. Solids 288, 81 (2001).

${ }^{6}$ D. R. Vollet, D. A. Donatti, A. Ibañez Ruiz, and W. C. de Castro, J. Non-Cryst. Solids 332, 73 (2003).

${ }^{7}$ S. J. Gregg and K. S. W. Sing, Adsorption, Surface Area and Porosity (Academic, New York, 1967).

${ }^{8}$ D.W. Schaefer and K.D. Keefer, Phys. Rev. Lett. 53, 1383 (1984).

${ }^{9}$ R. Vacher, T. Woignier, J. Pelous, and E. Courtens, Phys. Rev. B 37, 6500 (1988).

${ }^{10}$ G. Porod, in Small Angle X-ray Scattering, edited by O. Glatter and O. Kratky (Academic, London, 1982).

${ }^{11}$ G. Leofanti, M. Padovan, G. Tozzola, and B. Venturelli, Catal.
Today 41, 207 (1990).

${ }^{12}$ G.W. Scherer, D.M. Smith, and D. Stein, J. Non-Cryst. Solids 186, 309 (1995).

${ }^{13}$ G.W. Scherer, J. Non-Cryst. Solids 225, 192 (1998).

${ }^{14} \mathrm{Ph}$. Dieudonne and J. Phalippou, J. Sol-Gel Sci. Technol. 14, 249 (1999).

${ }^{15}$ T. Woignier, J. Phalippou, R. Vacher, J. Pelous, and E. Courtens, J. Non-Cryst. Solids 121, 198 (1990).

${ }^{16}$ P.W. Schmidt and Xie Dacai, Phys. Rev. A 33, 560 (1986).

${ }^{17}$ P. Pfeifer and D. Avnir, J. Chem. Phys. 79, 3558 (1983).

${ }^{18}$ A.B. Jarzȩbski, J. Lorenc, Y.I. Aristov, and N. Lisitza, J. NonCryst. Solids 190, 198 (1995).

${ }^{19}$ F. Ehrburger-Dolle, M. Holz, C. Mauzac, J. Lahaye, and G.M. Pajonk, J. Non-Cryst. Solids 145, 185 (1992).

${ }^{20}$ G. Schuck, W. Dietrich, and J. Fricke, in Aerogels, Springer Proc. in Physics Vol. 6, edited by J. Fricke (Springer, Berlin, 1986), p. 148.

${ }^{21}$ F. Ehrburger-Dolle, P.M. Mors, and R. Jullien, J. Colloid Interface Sci. 147, 142 (1991). 